\title{
Integrated Structural Sizing Optimization
}

\author{
Marc Hoit and Alfredo Soeiro \\ Department of Civil Engineering, University of Florida, Gainesville, FL 32601, USA
}

\section{Summary}

A method for structural member sizing optimization to be used for designing three dimensional structures is presented. The procedure is based on the Augmented Lagrangian Multiplier method. The algorithm includes the displacements as state variables and consequently combines the analysis and the search for the optimal section properties into a single step. This combination process avoids the usual cycling between analysis and optimization phases. The width and height of rectangular elements are chosen from a finite compact set. The static equilibrium equations are treated as equality constraints eliminating the need to solve the standard structural analysis problem. The method handles displacement and stress limits as inequality constraints.

\section{Solution Procedure}

A general nonlinear constrained optimization problem in the standard form may be described as finding the set of $\mathbf{n}$ design variables, contained in the vector $x$, such that the objective function is minimized, subject to constraints. Generally, in structural sizing optimization problems, the vector $X$ contains the physical dimensions of the elements: area and moments of inertia or equivalent type properties like height and width. The constraints represent the upper and lower bounds of physical dimensions, limits on the stresses on the structural elements, allowable displacements, buckling formulas and any other type of serviceability or structural performance demands.

The basis for the updated formulation is the inclusion of the global displacements and thus the equilibrium equations directly into the optimization formulation. By adding the displacements as unknowns and using the equilibrium equations 
as equality constraints in the optimization process, cycling between analysis and design is eliminated. This also creates a general formulation for any type of finite element optimization since the stiffness matrices are used directly. Through converting any constraint into its equivalent displacement formulation, we are not restricted to displacement constraints. This mapping into displacement space is a standard process used in all stiffness based analysis programs.

By formulating the problem in the displacement space, all classical optimization formulations are directly applicable since flexibility and stiffness are inverses of each other and displacements are unknowns instead of their counter part forces $[1,2,5]$. This direct use of the stiffness matrix allows existing finite element subroutines to be used directly with only the minor modification of taking the element properties from the state variable vector. It must be emphasized that the inclusion of displacements as variables does not increase the size of the problem since classical methods must also solve the analysis problem.

A classical solution approach to this type of mathematical problem with equalities and inequalities is through the formulation of a penalty function that includes the different functions of the variables. It is the Augmented Lagrangian multiplier method that is used as the penalty function for the solution process $[4,6]$. The use of this function replaces the constrained nonlinear problem with an unconstrained nonlinear problem. The Lagrangian multipliers are changed at the end of each unconstrained minimization cycle and are done according to the rules proposed by schult and Hestenes [6].

\section{Unconstrained Minimization}

The unconstrained problem has been solved by a variety of solution techniques [7]. In a previous version, the Hooke and Jeeves solution technique with pattern search was used [3]. To improve this crucial stage of the process, it was decided to replace that technique by the Conjugate Direction method of Fletcher and Reeves [7]. This is a variation of the steepest 